\section{Alloreactive Natural Killer cells have anti-tumor capacity against disseminated human multiple myeloma in Rag2-/- $\gamma$ C-/-mice when combined with low dose cyclophosphamide and total body irradiation}

\section{Subhashis Sarkar ${ }^{1}$, Willy Noort ${ }^{2,3}$, Catharina HMJ Van Elssen ${ }^{1}$, Richard Groen ${ }^{2,3}$, Louis van Bloois ${ }^{4}$, Michel van Gelder ${ }^{1}$, Harry C Schouten ${ }^{1}$, Marcel GJ Tilanus ${ }^{5}$, Wilfred TV Germeraad $^{1}$, Lotte Wieten ${ }^{5}$, Anton CM Martens ${ }^{2,3,6}$ and Gerard MJ Bos ${ }^{1 *}$}

${ }^{1}$ Department of Internal Medicine, Division of Hematology, Maastricht University Medical Center, Maastricht, The Netherlands

${ }^{2}$ Department of Cell Biology, University Medical Center Utrecht, Utrecht, The Netherlands

${ }^{3}$ Department of Hematology, VU University Medical Center, Amsterdam, The Netherlands

${ }^{4}$ Department of Pharmaceutical Sciences, Utrecht University, The Netherlands

${ }^{5}$ Department of Transplantation Immunology, Maastricht University Medical Center, Maastricht, The Netherlands

${ }^{6}$ Department of Immunology, University Medical Center Utrecht, Utrecht, The Netherlands

\begin{abstract}
Immunotherapy with alloreactive Natural Killer (NK) cells could be a new treatment option for the incurable plasma cell malignancy Multiple Myeloma (MM). In the present study, we explored the anti-MM potential of alloreactive NK cells using the RAG2-/- $\gamma \mathrm{c}-/-\mathrm{im}$ munodeficient disseminated mouse model. In this model, we studied NK cell-mediated Graft versus MM (GvM) responses while

*Corresponding author: Gerard MJ Bos, Department of Internal Medicine, Division of Hematology, Maastricht University Medical Center (MUMC) PO Box 5800, 6202 AZ Maastricht, The Netherlands, Tel: +31 433877026; Fax: +31 433875006; E-mail: Gerard.Bos@mumc.nl

Citation: Citation: Sarkar S, Noort W, Van Elssen CHMJ, Groen R, van Bloois $\mathrm{L}$, et al. (2015) Alloreactive Natural Killer cells have anti-tumor capacity against disseminated human multiple myeloma in rag2-/- $\gamma \mathrm{c}-/$-mice when combined with low dose cyclophosphamide and total body irradiation. J Clin Immunol Immunother 2: 008.
\end{abstract}

Received: July 06, 2015; Accepted: August 14, 2015; Published: September 02, 2015 concurrently examining the risk of developing Graft versus Host Disease (GvHD). First, luciferase expressing human U266 MM cells were intravenously (i.v.) injected in RAG2-/- $\gamma \mathrm{c}-/-$ mice, and human KIR-ligand alloreactive NK cells or PBMC were injected (i.v.) after Bioluminescent Imaging (BLI)-confirmed tumor formation. BLI of tumor growth kinetics in a preliminary experiment revealed that infusion of resting NK cells decreased MM burden in one out of four treated mice receiving 3 injections of $8 \times 10^{6}$ NK cells, with no signs of GvHD. Although infused PBMCs efficiently eradicated established MM in all treated mice, this concurrently induced lethal GvHD. rhIL-2 activated NK cells, injected in subcutaneously (s.c.) growing MM tumors, proved to be superior to resting NK cells and anti-MM responses occurred NK cell dose dependently. Since MM is a dispersed malignancy spread throughout the body, we investigated the therapeutic potential of rhIL-2 activated NK cells, from either cord blood or peripheral blood origin, in the disseminated MM mouse model. Whereas activated NK cells were unable to trigger efficient GvM responses when given alone, the infusion of activated NK cells following a non-curative conditioning treatment with cyclophosphamide and total body irradiation, did prolong progression free survival $(p=0.003)$ without any signs of GvHD. This study illustrates the therapeutic potential and safety of NK cell therapy in MM and is an important step towards cell-based immunotherapy for $\mathrm{MM}$ patients. It also establishes the RAG2-/- $\gamma \mathrm{C}-/-$ MM model as a clinically relevant pre-clinical model to further develop NK cell immunotherapy.

Keywords: Cancer; Immunotherapy; NK cell; Multiple Myeloma

\section{Introduction}

Multiple Myeloma (MM) is a hematological malignancy characterized by clonal expansion of plasma cells in the bone marrow [1]. Despite new options, state-of-the-art treatment results only in a median survival of 5-7 years [2]. New treatment strategies with curative potential with reduced toxicity are therefore urgently needed. Autologous-Stem Cell Transplantation (auto-SCT) combined with high dose chemotherapy has improved overall survival when compared to treatment with conventional chemotherapy alone; still the great majority of patients eventually relapse [3]. Allogeneic-SCT (allo-SCT) has the potential to cure MM by the so-called graft versus tumor response of donor immune cells. However, an important drawback of allo-SCT is the high risk of life-threatening Graft-versus-Host Disease (GvHD) mediated by donor $\mathrm{T}$ cells responding to mismatched histocompatibility antigens [4,5]. Moreover, treatment related mortality after allo-SCT is high, and patients surviving the procedure still show progression of disease in the majority of cases.

When allo-SCT is performed over HLA barriers, both Natural Killer (NK) cells and T cells can mediate GvM responses, but an important advantage of NK cells is that they do not seem to mediate GvHD [6]. NK cells are capable of targeting virally infected or malignantly transformed cells and become activated by tilting the delicate balance of inhibitory and activating receptor signals [7]. An important class of inhibitory receptors is the Killer Immunoglobulin-like Receptor (KIR) family interacting with the highly polymorphic HLA class I molecules expressed by virtually every healthy cell. The most important inhibitory KIRs are KIR3DL1 
Citation: Sarkar S, Noort W, Van Elssen CHMJ, Groen R, van Bloois L, et al. (2015) Alloreactive Natural Killer Cells Have Anti-Tumor Capacity Against Disseminated Human Multiple Myeloma in Rag2-/- $\gamma$ C-/-Mice When Combined With Low Dose Cyclophosphamide and Total Body Irradiation. J Clin Immunol Immunother 2: 008.

that binds HLA-Bw4 epitopes, KIR2DL2 and KIR2DL3 that interact with HLA-group C1 alleles and KIR2DL1 that binds HLA-group C2 alleles [8-10]. Upon engagement of inhibitory KIRs with the HLA class I molecules of a target cell, suppressive signals are transmitted, prohibiting NK cells to kill the target cell. This mechanism of self-recognition can be used in anti-tumor therapy, by transplantation of NK cells with a licensed KIR repertoire that mismatches for the donor HLA according to the "missing self" principle $[11,12]$. Ruggeri et al., showed that the anti-tumor effect KIR-mismatched haplo-identical SCT setting in Acute Myeloid Leukemia (AML) is attributed to the presence of "alloreactive NK cells" [6]. AML patients treated with a KIR-ligand mismatched allogeneic bone marrow transplant show increased survival over patients transplanted without such a mismatch [13].

There is accumulating evidence to support a role for NK cells in controlling the progression of MM [14-20]. Therapeutic interventions like lenalidomide and thalidomide increase NK cell reactivity and thereby improve survival. Additionally, there is evidence that alloreactive NK cells may be relevant in MM. In a group of 10 patients with MM that were treated with alloreactive NK cells, fifty percent demonstrated a response, with some patients having a complete remission [18]. Recently, these authors expanded their observations in more patients, showing that freshly prepared NK cells could continue to expand in vivo after infusion, but only in two of eight patients a clinical response could be attributed to the infused NK cells [19]. Kroger et al., analyzed patients that were transplanted with an unrelated donor, which included donors with a mismatch for HLA-C groups, thereby allowing NK cell alloreactivity. The group of patients that received a transplant with the alloreactive NK cells indeed showed a far lower incidence of disease recurrence [21].

Unfortunately, since MM is a disease of the elderly, the majority of MM patients is not eligible for KIR mismatched haplo-identical SCT due to the severe conditioning regime and transplantation related complications and thus indicating that other modalities have to be developed. Our previous research lead us to the idea that infusion of only KIR-ligand mismatched NK cells is an interesting treatment option to provide curative effects that bypasses the risks that are associated with a full HLA mismatched allo-SCT. In vitro, NK cells have been shown to efficiently kill MM cells $[16,17]$. Recently, we showed that KIR-ligand mismatched NK cells are better in doing this than matched cells [22]. We could demonstrate that it is also possible to kill MM cells under hypoxia, which is an immunosuppressive condition relevant in this disease [23]. Before performing clinical trials, immunodeficient mouse models provide an excellent opportunity to test novel treatment strategies. The $\mathrm{RAG}^{-/-} \gamma \mathrm{c}^{-/} \mathrm{MM}$ mouse model was originally developed by some of us to study GvHD development after injection of PBMCs or purified $\mathrm{T}$ cells [24]. In this model, we previously showed that human PBMC xenografts did not only induce GvHD but also GvM [25]. In the present study, we used this $\mathrm{RAG}^{-1-} \gamma \mathrm{c}^{-1} \mathrm{MM}$ mouse model to obtain the proof of concept for the effect of alloreactive KIR-ligand mismatched NK cells on GvM and GvHD in MM bearing mice.

\section{Materials and Methods}

\section{Cell lines and cell cultures}

The human MM cell lines studied were U266, RPMI-8226/S, UM-9, LME-1, XG-1, OPM-1, and L-363, all retrovirally transduced with the luciferase gene as described previously [20]. U226 had been transfected with GFP previously. All MM cell lines were cultured in RPMI-1640 (Gibco, Breda, The Netherlands) except for XG-1 and LME-1, which were cultured in IMDM medium (Gibco), supplemented with $10 \%$ fetal calf serum (Integro, Zaandam, The Netherlands), $100 \mathrm{U} / \mathrm{ml}$ penicillin (Gibco), $100 \mu \mathrm{g} / \mathrm{mL}$ streptomycin (Gibco). The XG-1 cell line was additionally cultured in the presence of $20 \mu \mathrm{g} / \mathrm{ml}$ Transferin (Sigma) and $500 \mathrm{pg} / \mathrm{ml} \mathrm{IL-6} \mathrm{(Biosource).}$ The cultures were maintained at $37^{\circ} \mathrm{C}$ with $5 \% \mathrm{CO}_{2}$ in humidified atmosphere. Presence and absence of HLA epitopes (HLA-C1, -C2 and -Bw4) and of HLA-A in cell lines (Table 1) and healthy control donors, were routinely determined by Sequence-Specific Oligonucleotides (SSO) analysis and Luminex ${ }^{\circledR}$ according to manufacturer's guidelines (One Lambda, Montpelier, France).

\begin{tabular}{|c|c|}
\hline Cell line & HLA genotype \\
\hline U266 & C1+ C2- Bw4- \\
\hline L363 & C1+ C2- Bw4- \\
\hline LME-1 & C1+ C2- Bw4- \\
\hline UM-9 & C1+ C2- Bw4- \\
\hline RPMI-8226/s & C1+ C2+ Bw4- \\
\hline OPM-1 & C1+ C2+ Bw4- \\
\hline XG-1 & C1+ C2+ Bw4+ \\
\hline
\end{tabular}

Table 1: Matched and mismatched KIRs based on genotypic expression of HLA epitopes. Genotypic expression of HLA-class I epitopes as determined by luminex-SSO.

\section{Mice}

The $\mathrm{RAG}^{-/} \gamma \mathrm{c}^{-/}$mice were originally obtained from the Amsterdam Medical Center (Amsterdam, The Netherlands). They were bred and maintained in filter top cages under specified pathogen-free conditions at the Central Laboratory Animal Institute (Utrecht University, Utrecht, The Netherlands) and received sterile water and irradiation sterilized food pellets ad libitum. All animal experiments were approved by the Ethical Committee for Animal Experimentation of Utrecht University and in accordance with current Dutch Law on Animal Experiments.

\section{Generation of Multiple Myeloma (MM) RAG2 $2^{-/-} \gamma \mathrm{c}^{-/-}$mice}

Generation of MM in RAG2 $2^{-1} \gamma \mathrm{c}^{-1}$ mice was performed as described in more detail in [25]. In brief, twenty-four hours before the injection of freshly cultured MM cells, mice received total body irradiation conditioning treatment (TBI; $3.0 \mathrm{~Gy}, 200 \mathrm{kV}$ X-rays). $5 \times 10^{6} \mathrm{U} 266$ cells were injected intravenously (i.v.) via the lateral tail vein. For the experiments with subcutaneous (s.c.) MM tumors, $0.3 \times 10^{6}, 1 \times 10^{6}$, $3 \times 10^{6}$ or $9 \times 10^{6} \mathrm{U} 266$ cells in $100 \mu \mathrm{l}$ phosphate-buffered saline were injected s.c. in the flank of the mice. In all experiments, tumor load was determined by Bioluminescence Imaging (BLI) measurements (Biospace $\Phi$-Imager and Acquisition software) on the ventral and dorsal sides of the mice. The BLI images were analyzed with M3Vision software (Biospace, Paris, France). In case of paralysis of the hind limbs or when the mice became moribund, they were sacrificed by cervical dislocation. To determine progression free survival mean BLI signal (ventral BLI signal + dorsal BLI signal)/2 for each time point was calculated. Progression was defined as a mean BLI signal that was greater than $2 \mathrm{x}$ the mean BLI signal at start of treatment on day 34 when applicable. 
A

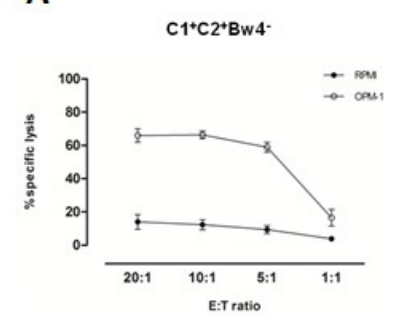

B
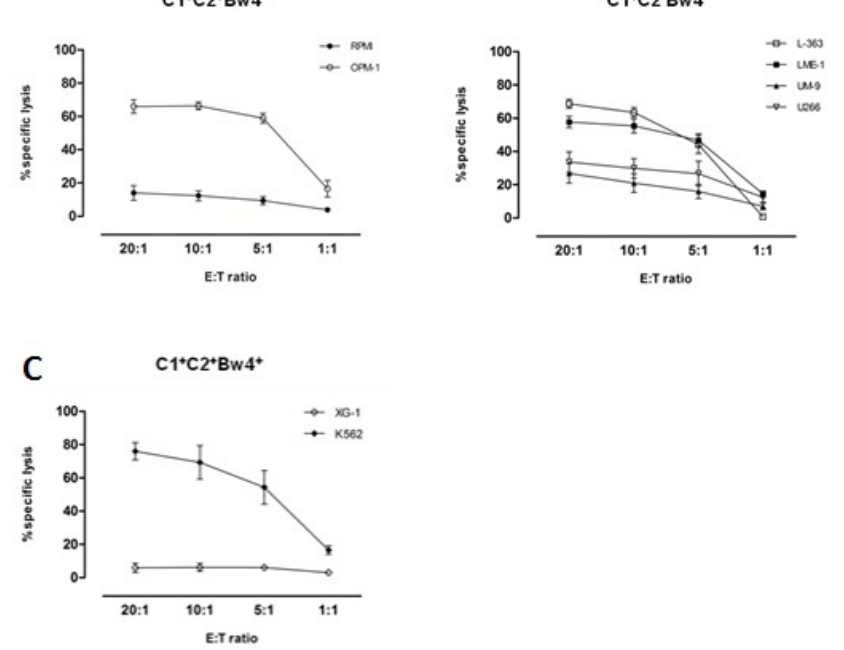

Figure 1: Alloreactive NK cells kill MM cell lines in vitro.

NK cells were isolated from healthy donors positive for all three KIR epitopes (HLA-C1, -C2, and -Bw4) and were co-cultured with DiO labeled MM cells at 20:1, 10:1 5:1 and 1:1 effector:target ratio. After 12 hours, the percentage of death (PI positive) MM cells was determined by flow cytometry. Graphs depict means and SD of specific lysis by NK cells from six different donors. A) Specific lysis of MM cell lines expressing HLA-C1 and -C2 but not Bw4 epitopes, B) MM cell lines expressing HLA-C1 but not $\mathrm{C} 2$ and Bw4 epitopes and C) MM cell line expressing all three NK cell epitopes.

\section{Preparation and transplantation of human PBMCs and peripheral blood derived NK cells}

Buffy coats were obtained from healthy human blood donors that were HLA-genotyped (all donors were HLA-C1, C2 and Bw4 positive) and KIR genotyped (all donors expressed KIR2DL1, KIRDL2/3 and KIR3DL1) by Sequence-Specific Oligonucleotides (SSO) analysis and Luminex ${ }^{\circledR}$ according to manufacturer's guidelines (One Lambda). Mononuclear cells from peripheral blood of these healthy donors were isolated by density gradient separation. NK cells were either negatively (for NK cell groups) or positively (for NK-depleted group) selected by immunomagnetic cell separation according to manufacturer's protocol (Miltenyi Biotec $\mathrm{GmbH}$, Bergisch Gladbach, Germany). The purity of isolated populations typically exceeded $95 \%$ as determined by flow cytometry. Unactivated NK cells were used directly upon isolation. For the experiments with IL-2 activated NK cells, the isolated NK cells were cultured overnight with $1000 \mathrm{U} / \mathrm{ml}$ of IL-2 (Proleukin). Cord blood derived NK cells were expanded and differentiated into NK cells from umbilical cord blood CD34+ cells (HLA-C1, C2 and Bw4 positive) as described before $[26,27]$ and were kindly provided by Dr. Spanholz and Dr. Groenewegen (Glycostem, Nijmegen, The Netherlands). Before injection, cells were counted and resuspended at the concentration indicated in the results section and legends. Suspensions of PBMC or NK cells were injected in a volume of $0.2 \mathrm{ml}$ either intravenously into the mice via the tail vein or intratumorally. Mice received $0.2 \mathrm{ml}$ of the liposomal suspension intravenously one day before injection of the cells. Control mice received liposomes as well but no PBMC or NK cell infusion. Liposomes were prepared as described previously [25]. GvHD was scored (4-point scale) on the parameters poor physical condition, ruffled fur, arched back, and a body weight loss greater than 10 percent.

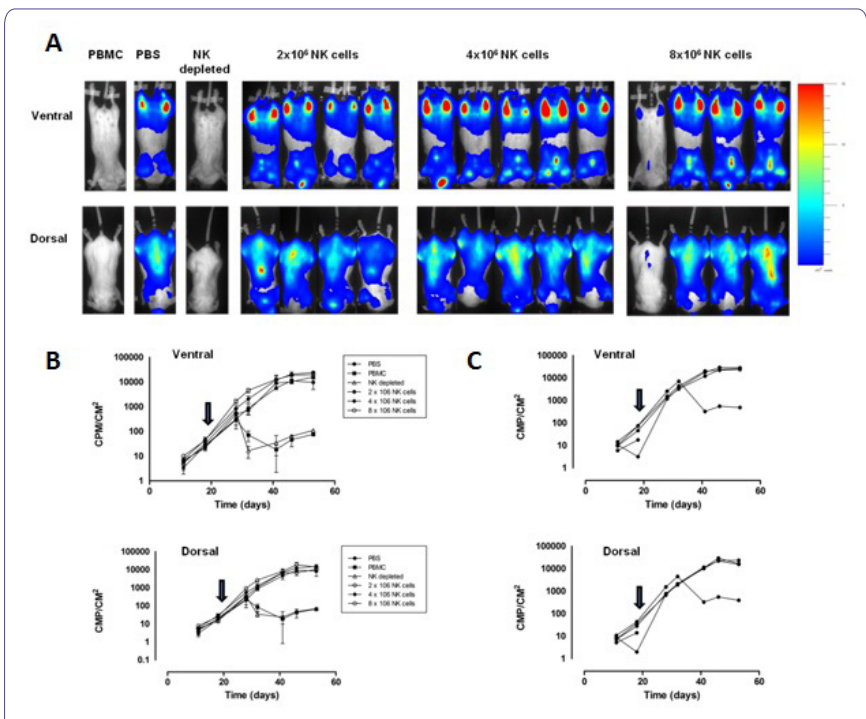

Figure 2: Freshly isolated, resting allogeneic NK cells can mediate anti-MM effects in vivo.

At day $0,5 \times 10^{6} \mathrm{U} 266$ cells were i.v. injected into RAG2-/ mice received one injection with liposomes followed on day 21,23 and 25 by i.v. injection of either $8 \times 10^{6} \mathrm{PBMC}$ or freshly isolated NK cells $\left(2 \times 10^{6}, 4 \times 10^{6}\right.$ or $8 \times 10^{6} \mathrm{NK}$ cells per injection). One group received three injections with NK cell depleted PBMC. PBMC and NK cells were obtained from donors expressing all three KIR ligands (i.e., HLA-C1, -C2 and -Bw4). Tumor growth was monitored by $\mathrm{BLI}$ of the ventral and dorsal side. A) BLI images obtained on day 53 of representative mice of the control, PBMC and NK cell depleted PBMC groups and of individual mice of the NK cell groups. B) Graphs show per treatment group mean and SD of the BLI signal measured on the indicated days. C) Graphs show BLI data per mouse for the group receiving three injections of $4 \times 10^{6} \mathrm{NK}$ cells. Arrows in the graphs in $\mathrm{B}$ and $\mathrm{C}$ indicate the time of PBMC or NK cell infusion.

\section{Flow cytometry}

Cell isolates from hind limbs were analyzed using flow cytometry for the presence of human cells in the total nucleated cell fraction. Bone marrow was obtained by flushing the bones with PBS. Single cell suspensions from the soft tissues were obtained by passaging through $70 \mu \mathrm{m}$ cell strainers (Becton Dickinson Biosciences, Erembodegem, Belgium). Fluorochrome conjugated monoclonal antibodies specific for surface molecules were used: human-CD3, human-CD45, mouse-CD45, human-CD56 all obtained from BD Biosciences. Cells were incubated with antibodies at proper dilutions for 30 minutes on ice. Samples were analyzed on a FACS Canto II (BD) with FACS Diva (BD) software.

\section{NK cell Cytotoxicity assay}

Enriched NK cells, isolated as described in the preparation and transplantation of huPBMCs section, were used in cytotoxicity assays. Target cells (MM cells) were labeled with 3'-Dioctadecyloxacarbocyanine (DiO) according to the manufacturer's instructions (Sigma) and $2 \times 10^{4}$ target cells were incubated with NK cells at various Effector:Target (E:T) ratios for 12 hours, each ratio in triplicate. Percentage of killed target cells (\% PI positive and $\mathrm{DiO}$ positive cells) was determined by flow cytometry. Percentages specific lysis was calculated as follows: ((\% PI positive target cells - \% spontaneous PI positive cells)/ \% of viable cells $) \times 100$. 


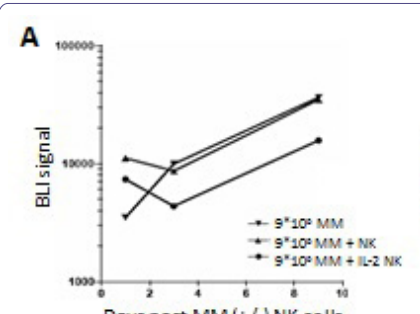
Days post MM (+/-) NK cells
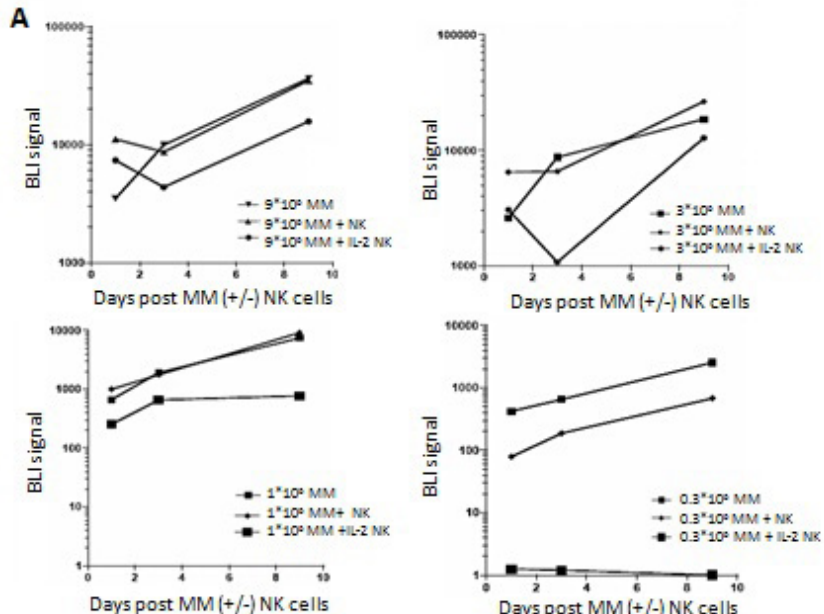

Days post MM $(+/-)$ NK cells

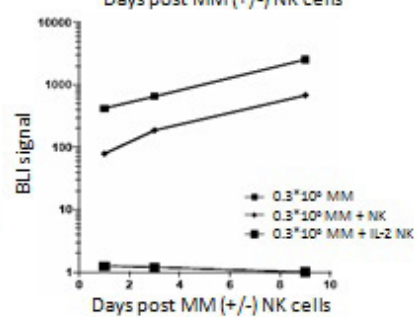

B

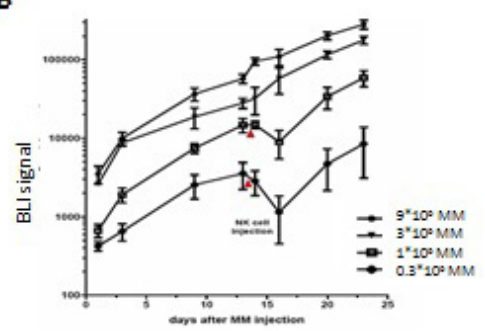

Figure 3: IL-2 activated NK cells mediate anti-MM responses in vivo in a dose dependent manner.

A) U266 MM cells $\left(0.3 \times 10^{6}, 1 \times 10^{6}, 3 \times 10^{6}\right.$ or $9 \times 10^{6}$ U266 cells) were mixed with $1 \times 10^{6} \mathrm{NK}$ cells. NK cells were either freshly isolated or activated overnight with $1000 \mathrm{U} / \mathrm{ml}$ of IL-2 (Proleukin). Immediately after mixing cells were injected s.c. in the flank of RAG2-/-/- mice. Control mice received U266 cells without NK cells. Tumor growth was monitored on the indicated days by BLI. Graphs show the mean BLI signal of $n=3$ mice per treatment group (no NK cells, unactivated, resting NK cells and activated NK cells). Each graph depicts one dose of U266 cells. B) RAG2-/ 2 -/-mice were s.c. injected with either $0.3 \times 10^{6}, 1 \times 10^{6}, 3 \times 10^{6}$ or $9 \times 10^{6} \mathrm{U} 266$ cells. NK cells were overnight activated with $1000 \mathrm{U} / \mathrm{ml} \mathrm{IL-2}$ and were injected intratumorally on day 13 . Tumor growth was monitored at the indicated days by BLI. Graph shows mean BLI signal per group of $n=3$ mice. Arrow indicates the time of NK cell injection.

\section{Statistics}

Differences in progression free survival (Figure 1 and 2) were calculated with Graphpad Prism software version 5.01 using log rank test (Mantel Cox) test.

\section{Results}

\section{Alloreactive NK cells kill MM cell lines in vitro}

To test whether freshly isolated alloreactive KIR-ligand mismatched NK cells could eliminate MM cells in vitro, NK cell alloreactivity against a panel of eight $\mathrm{MM}$ cell lines was determined. These cell lines were first genotyped for HLA-A,-B and-C to determine which KIR ligands were present and absent. Two of the cell lines, i.e., RPMI-8226/s and OPM-1, expressed HLA-C1 and -C2 but not the Bw4 epitope (Table 1). U266, LME-1, L-363 and UM-9 expressed only the HLA-C1 and not the C2 and Bw4 epitopes. XG-1 was the only cell line expressing all three epitopes. Then, cell lines were co-cultured with freshly isolated NK cells derived from healthy donors. To avoid hyporesponsiveness of NK cells due to lack of licensing, donors expressing all three epitopes (i.e., positive for HLA-C1, -C2 and -Bw4) were used. After 12 hours of co-culture, the
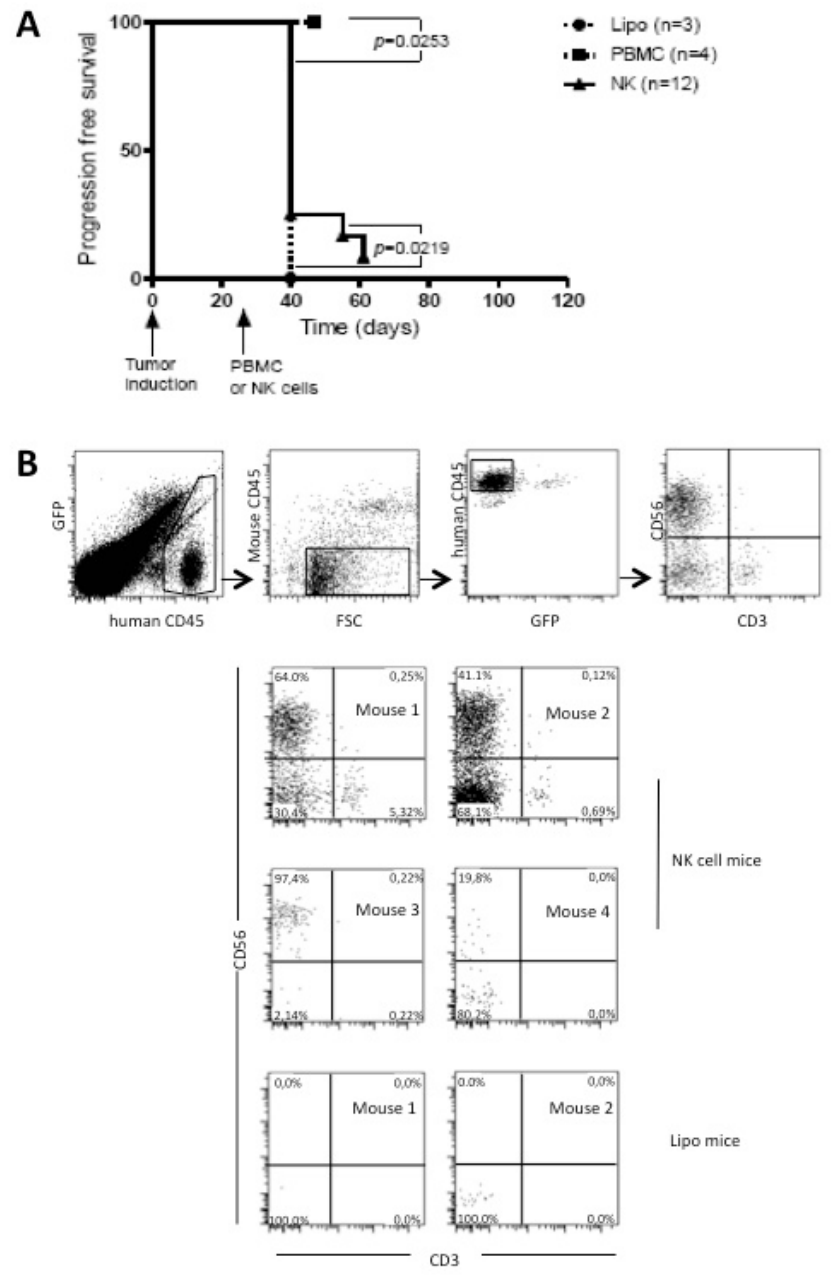

Figure 4: IL-2 activated NK cells can home to the bone marrow of MM bearing RAG2-/E-/- mice.

At day $0,5 \times 10^{6}$ U266 cells were i.v. injected into RAG2-/ 20 mice received one injection with liposomes followed on day 21, 23 and 25 by i.v. injection of $8 \times 10^{6}$ PBMC or NK cells that were overnight activated with $1000 \mathrm{U} / \mathrm{ml} \mathrm{IL-2}$. Tumor growth was monitored by BLI of the ventral and dorsal side. A) Graph shows progression free survival per treatment group (days). To determine progression free survival the mean BLI signal (ventral BLI signal + dorsal BLI signal)/2 for each time point was calculated. Progression was defined as a mean BLI signal that was greater than $2 x$ the mean BLI signal at day 34. B) Bone marrow was obtained at the day of sacrifice of the mice. Homing of human leucocytes to the bone marrow was analyzed by flow cytometry. Plots show representative gating strategy of human CD45+ cells, mouse CD45- cells, GFP- cells (since MM cells are transduced with GFP) and examples of $n=4 N K$ cell treated mice and $n=2$ control mice receiving only liposomes but not NK cells or PBMC. GFP = Green fluorescent protein. FSC = Forward Scatter.

percentage of death MM cells was determined by flow cytometric analysis of the percentage PI positive MM cells. This revealed that killing of all MM lines and of K562, a leukemia cell line devoid of HLA class I expression and used as positive control cell line for NK mediated lysis, occurred in a NK cell concentration dependent manner (Figure $1 \mathrm{~A}-\mathrm{C}$ ). Killing efficiency differed between the lines; LME-1, L363, OPM-1 were almost as sensitive to NK cell killing as K562 (Figure $1 \mathrm{~A}-\mathrm{B}$ ) whereas the $\mathrm{C} 1+\mathrm{C} 2+\mathrm{Bw} 4+$ cell line $\mathrm{XG}-1$ was quite resistant to killing by alloreactive NK cells (Figure 1C). Together this shows that MM cell lines, though at different efficiencies, can by killed by alloreactive NK cells in vitro. 

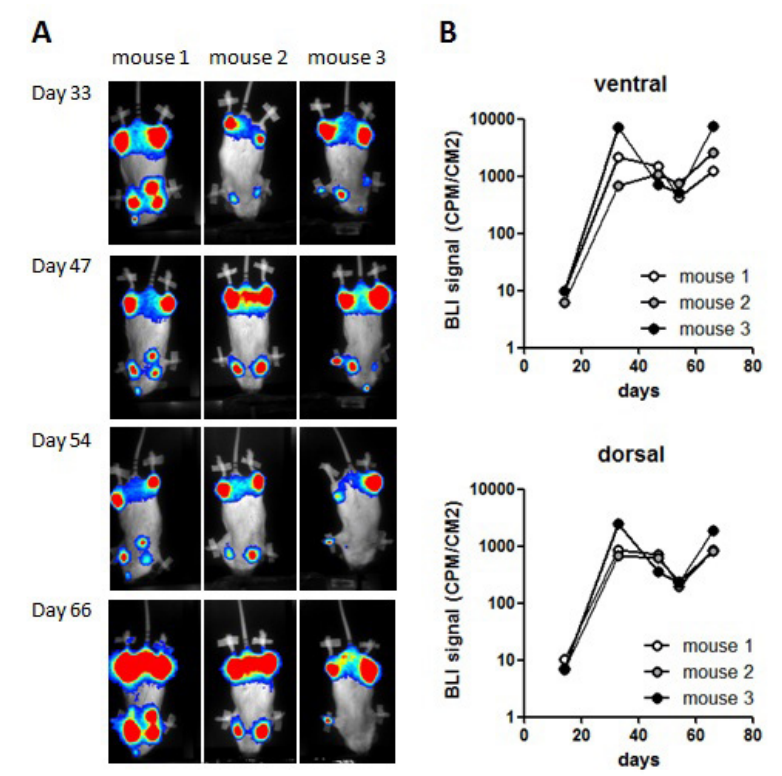

Figure 5: Cyclo/TBI treatment alone does not cure disseminated MM.

RAG2-/ $/$-/-mice were i.v. injected with $5 \times 10^{6}$ U266 cells on day 0 . On day 34 and 35 , mice were irradiated with 2 Gy. On day 35, mice also received an injection with $50 \mathrm{mg} / \mathrm{kg}$ cyclophosphamide. Tumor growth was monitored by $\mathrm{BLI}$ on both ventral and dorsal sides. A) Dorsal BLI images from the mice at the indicated days. B) Graph shows BLI signal per mouse on the indicated days.

Freshly isolated, resting alloreactive NK cells can mediate anti-MM effects in vivo

To investigate whether NK cells mediate anti-MM responses in vivo, the $\mathrm{RAG}^{-1-} \mathrm{\gamma c}^{-1-}$ mouse disseminated $\mathrm{MM}$ model was used with the U266 cell line. This cell line was killed by NK cells in vitro (Figure 1), and in a previous study we showed that infusion of PBMC could cure mice from established MM induced by injection of U266 cells [25]. In the current study, $5 \times 10^{6}$ U266 cells were i.v. injected on day 0 followed by i.v. injection of either PBMC or freshly isolated, resting NK cells at different doses on day 21,23 and 25 . To test whether NK cells were required for the anti-tumor response after PBMC injection, one group of mice received PBMC depleted from NK cells. Both PBMC and NK cells were obtained from peripheral blood of donors expressing all three KIR ligands (i.e., HLA-C1, -C2 and $-\mathrm{Bw} 4)$. Monitoring of tumor growth by BLI revealed that all treatment groups had developed $\mathrm{MM}$ at the day of PBMC or NK cell injection (Figure $2 \mathrm{~A}-\mathrm{B}$ ). As expected from our previous studies, injection of PBMC subsequently completely cured the mice from MM. NK cells were not required for this curative effect as injection of NK cell depleted PBMC was as effective as injection of the complete PBMC fraction. NK cell treatment was performed by three times injection (days 21,23 and 25) with either $2 \times 10^{6}, 4 \times 10^{6}$ or $8 \times 10^{6} \mathrm{NK}$ cells per injection. In none of the two groups receiving the lower NK cell doses this resulted in a robust anti-MM response and tumor load of the NK cell groups was comparable to the tumor load in the control group (i.e., group w.o. PBMC or NK cells; Figure 2 A-B). However, in the group receiving the highest dose of $\mathrm{NK}$ cells one mouse apparently showed a reduction in tumor burden upon infusion of NK cells hinting that purified NK cells can mediate anti-MM responses in this model (Figure 2C). In the groups receiving PBMC or NK cell depleted PBMC all mice developed signs of GvHD as observed by visual inspection of the mice and by weight loss. Importantly, none of the NK cell treated mice showed signs of GvHD demonstrating the safety of NK cell infusion.

\section{IL-2 activated NK cells mediate anti-MM responses in a dose dependent manner in vivo}

NK cells can eliminate MM cells in vitro, however in vivo no anti-myeloma effect is seen. Therefore, we hypothesized that the bone marrow micro-environment could suppress NK cell function. To test if NK cells were actually able to function in the RAG2 $2^{-1-} \mathrm{cc}^{-1}$, we performed an in vivo kill assay. We have previously shown that IL-2 activation can potentiate the anti-MM response of NK cells in vitro. As in this case the number of NK cells needed for the in vivo kill is expected to be lower, U266 MM cells $\left(0.3 \times 10^{6}, 1 \times 10^{6}, 3 \times 10^{6}\right.$ or $9 \times 10^{6}$ U266 cells) were mixed with $1 \times 10^{6}$ IL-2 activated NK cells followed by immediate s.c. injection in the flank of RAG2 $2^{-/} \gamma \mathrm{c}^{-/-}$mice. Monitoring of tumor growth showed that the BLI signal increased between day 0 and day 9 in the groups of mice receiving any of the four doses of U266 cells but not NK cells (Figure 3A). This increase in BLI signal was also observed for the groups receiving U266 cells and non-activated, resting NK cells. For all groups injected with U266 cells and IL-2 activated NK cells the BLI signal was lower than that of the corresponding control groups and in the group receiving the lowest number of $\mathrm{U} 266$ cells the effect was already detectable by in vivo imaging on day 1. Co-injection of activated NK cells reduced outgrowth of the tumor showing NK cells are active in this model (Figure 3A).

The above-mentioned data demonstrated that the effect of IL-2 activated NK cells occurred in a dose dependent manner. To investigate whether IL-2 activated NK cells could eliminate established MM in vivo as well, mice were s.c. injected with either $0.3 \times 10^{6}, 1 \times 10^{6}, 3 \times 10^{6}$ or $9 \times 10^{6}$ U266 cells. Upon development of a s.c. tumor on day 13 , IL-2 activated NK cells were injected intratumorally. In the two groups receiving the highest number of U266 cells $\left(3 \times 10^{6}\right.$ or $9 \times 10^{6}$ cells), NK cell injection did not reduce the tumor load (Figure 3B). However, in the two groups receiving the lowest number of U266 cells $\left(0.3 \times 10^{6}\right.$ or $1 \times 10^{6}$ cells $)$ a reduction in the BLI signal could be observed after NK cell injection. Together these data demonstrate that IL-2 activated NK cells can mediate anti-MM responses in vivo but that the effector to target ratio is very important for in vivo responses.

\section{IL-2 activated human NK cells can home to the bone marrow of MM bearing RAG2 $2^{-/} \gamma \mathrm{c}^{-/-}$mice}

As we established that IL-2 activated NK cells are indeed effective in $\mathrm{RAG}^{-/-} \gamma \mathrm{c}^{-/ /}$mice after subcutaneous injection, we next investigated the effect of IL-2 activated KIR-ligand mismatched NK cells in the disseminated MM model. To this end, $5 \times 10^{6}$ U266 cells were i.v. injected, followed by i.v. injection of PBMC on day 21, 23 and 25, $\left(\mathrm{n}=4\right.$ mice) or $8 \times 10^{6} \mathrm{NK}$ cells that were overnight activated with IL-2 (on day 21, 23 and 25, $\mathrm{n}=6$ mice). As an alternative source of activated NK cells, we used NK cells expanded from cord blood and six mice received on day 21 one injection with $24 \times 10^{6}$ cord blood derived NK cells. Since it has previously been shown that IL-15 administration alone promotes in vivo survival and expansion of NK cells while it does not have an anti-tumor effect [28], IL-15 was injected i.p. three times a week for two weeks after NK cell infusion in this experiment. Around day 40, all control mice (mice receiving no NK cells or PBMC) were sacrificed due to high tumor burden. For the NK cell treated mice progression free survival was longer than for control mice $(\mathrm{p}=0.02$; Figure 4A). Nevertheless, also the NK cell treated mice died due to high tumor load. In line with our experiment in Figure 2, injection of PBMC reduced tumor burden but these mice had to be sacrificed 
Citation: Sarkar S, Noort W, Van Elssen CHMJ, Groen R, van Bloois L, et al. (2015) Alloreactive Natural Killer Cells Have Anti-Tumor Capacity Against Disseminated Human Multiple Myeloma in Rag2-/- $\gamma$ C-/-Mice When Combined With Low Dose Cyclophosphamide and Total Body Irradiation. J Clin Immunol Immunother 2: 008.

due to severe GvHD around day 50. Symptoms of GvHD were not observed in NK cell treated mice. Importantly, flow cytometric analysis of the bone marrow harvested at the day of sacrifice of the mice revealed that cells expressing the human leukocyte marker CD45 and CD56 but not CD3 (hCD45+CD56+CD3- is indicative of NK cells) were present in the bone marrow (Figure 4B). These hCD45+CD56+CD3- NK cells were only present the IL-2 activated NK cell treated groups indicating that NK cells could actually home to the bone marrow. In the mice receiving NK cells obtained from peripheral blood hCD45+CD56-CD3+ T cells (range $0-5.32 \%$ ) and cells expressing hCD45+ but not CD56 or CD3 were also present (Figure 4B) in the bone marrow.

\section{Cyclophosphamide (cyclo)/Total Body Irradiation treatment alone does not cure disseminated MM}

(TBI)

In parallel studies in a mouse model for breast cancer, we obtained evidence that cyclo/TBI conditioning is important for in vivo anti-tumor responses of alloreactive NK cells against $4 \mathrm{~T} 1$ breast cancer tumor cells [29]. In the current study, we therefore aimed to investigate whether addition of cyclo/TBI to the treatment regime resulted in prolonged progression free survival in the $\mathrm{RAG}^{-1-} \mathrm{\gamma c}^{-1}$ MM model. To confirm that cyclo/TBI treatment was performed at a dosage that by itself was not sufficient to eliminate the tumor, we
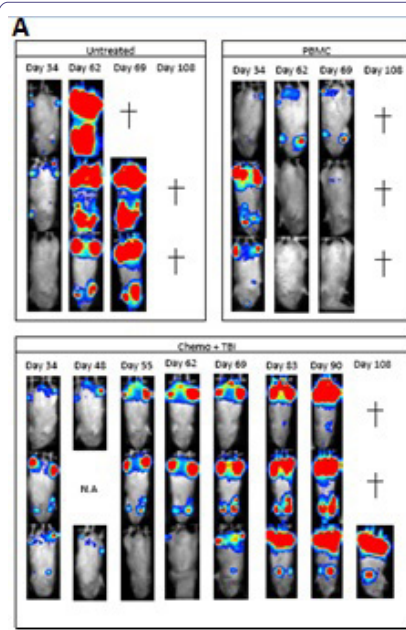

B

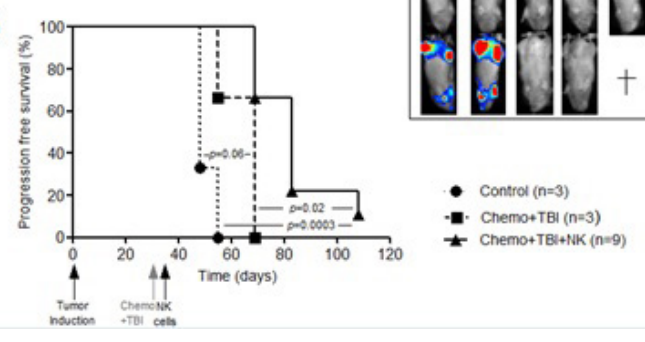

Figure 6: Combination treatment with cyclo/TBI and IL-2 activated NK cells provides anti-MM responses in the disseminated model.

RAG2-//-/mice were i.v. injected with $5 \times 10^{6}$ U266 cells on day 0 . On day 34 and 35 , mice were irradiated with 2 Gy. On day 35 , mice also received an injection with $50 \mathrm{mg} / \mathrm{kg}$ cyclophosphamide. NK cell treated mice also received activated $8 \times 10^{6} \mathrm{NK}$ cells (blood or cord blood derived) on days 36, 38 and 42 by i.v. injection. Control mice received neither cyclo/TBI nor NK cells. Tumor growth was monitored by BLI on both ventral and dorsal sides. A) Dorsal BLI images from the mice at the indicated days. B) Graph shows progression free survival per treatment group. To determine progression free survival means $\mathrm{BLI}$ signal (ventral BLI signal + dorsal BLI signal)/2 for each time point was calculated. Progression was defined as a mean BLI signal that was greater than $2 x$ the mean BLI signal at day 34 . performed a pilot experiment in which $\mathrm{RAG2}^{-/} \gamma \mathrm{c}^{-/}$mice were i.v. injected with $5^{\star} 10^{6} \mathrm{U} 266$ cells on day 0 . After development of MM, mice were irradiated with 2 Gy on day 34 and day 35 . On day 35 mice also received $50 \mathrm{mg} / \mathrm{kg}$ cyclophospamide. Monitoring of tumor growth revealed that although cyclo/TBI treatment initially reduced the tumor burden, it was not sufficient to cure the mice as tumors started growing again in these mice (Figure 5).

\section{Combination treatment with cyclo/TBI and IL-2 activated NK cells can provide anti-MM responses in the disseminated MM model}

To investigate the effect of treatment with IL-2 activated NK cells in combination with cyclo/TBI, MM was induced in RAG2 $2^{-1} \mathrm{\gamma c}^{-/}$mice by i.v. injection of $5 \times 10^{6} \mathrm{U} 266$ cells on day 0 . In line with our pilot, cyclo/TBI treatment was done at days 34 and 35 and tumor growth was monitored by BLI. This confirmed that cyclo/TBI treatment only slightly, and not significantly, increased progression free survival as compared to control mice ( $\mathrm{p}=0.06$; Figure 6$)$. However, chemo/radio therapy in combination with injection of $8 \times 10^{6} \mathrm{IL}-2$ activated blood derived NK cells (on day 36,38 and 42 ) or $24 \times 10^{6}$ of cord blood-derived NK cells on day 36 prolonged progression free survival as compared to control mice ( $\mathrm{p}=0.003$; Figure 6 ). No difference was noted between the two sources of NK cells. Two mice in the NK cell treated group died for unknown reason while having a very low tumor load and without having signs of GvHD. In the other NK cell treated mice we also did not observe signs of GvHD by visual inspection. Although MM eventually progressed, this shows that combination treatment with cyclo/TBI and IL-2 activated alloreactive NK cells are able to provide anti-MM effects against established disseminated MM.

\section{Discussion}

Despite novel treatment options in the last decade, MM remains largely incurable illustrating the need to develop novel therapeutic treatment options. In the present proof-of-concept study, we investigated the effect of alloreactive KIR-ligand mismatched NK cells on GvM and GvHD in the RAG2 $2^{-/} \gamma \mathrm{c}^{-/-}$mouse MM model. This revealed that IL-2 activated NK cells in combination with low dose conditioning with cyclo/TBI can mediate anti-MM responses. Importantly, we did not observe a single sign of GvHD in any of the experimental treatment protocols upon NK cell injection. In line with these data, a first clinical study in MM showed that KIR-ligand mismatched NK cells could be infused into MM patients without causing toxicity and GvHD [18] and comparable observations have been made in AML. Altogether, this illustrates the feasibility and clinical potential of the approach.

Thus far, the clinical efficacy of NK cell infusion in cancer patients has been somewhat inconsistent emphasizing that optimization of therapeutic protocols is required [30]. NK cells can kill only a limited number of target cells. In the current study, we observed that NK cell responses against s.c. MM occurred in a NK cell dose dependent manner (Figure 3) and with unactivated, resting NK cells, we possibly observed a biological effect of NK cells in one of the four mice (25\%) with disseminated MM in the group receiving the highest NK cell dose (Figure 2). Dose dependency has also been shown in the 5T33M MM model were the anti-MM response of transplanted mouse NK cells could be enhanced by infusion of a higher number 
Citation: Sarkar S, Noort W, Van Elssen CHMJ, Groen R, van Bloois L, et al. (2015) Alloreactive Natural Killer Cells Have Anti-Tumor Capacity Against Disseminated Human Multiple Myeloma in Rag2-/- $\gamma$ C-/-Mice When Combined With Low Dose Cyclophosphamide and Total Body Irradiation. J Clin Immunol Immunother 2: 008.

of NK cells [31]. In another study, with human NK cells expanded on K562-mb15-41BBL feeder cells, adoptive transfer of $160 \times 10^{6} \mathrm{NK}$ cells inhibited growth of OPM2 while transfer of $40 \times 10^{6} \mathrm{NK}$ cells was not sufficient [32]. These data suggest that infusion of really high numbers of NK cells are needed to improve clinical efficacy of NK cell therapy. Numbers that can be obtained using a bioreactor (our unpublished data).

In our model, mice having established MM were treated with NK cells. In that way, our model resembles the clinical situation were plasma cells are dispersed over multiple sites encompassing the entire skeletal system. Because of this high tumor load, a major caveat is the number of NK cells required for clinically effective therapy that can be obtained by isolation from peripheral blood. Ex vivo expanded NK cells could provide a solution and are an interesting alternative for NK cells derived from apheresis products. An additional advantage is that the ex vivo culturing period would allow for extra NK cell activation. In the current study, we used two sources of NK cells; IL-2 activated NK cells isolated from blood of healthy individuals and NK cells that were generated and expanded from cord blood [26,27]. With both sources we observed reactivity against established, disseminated MM in combination with chemo/TBI (Figure 6). This is in line with two previous studies showing that NK cells expanded on K562-mb 15-41BBL feeder cells [32] and the NK cell lines NK-92 or KHYG-1 [33] reduced disease burden in vivo (in mouse models for MM), together demonstrating that different sources of NK cells could be further tested for clinical application.

In the current study, NK cell donors were selected based on the genotypic presence of a mismatch between inhibitory KIRs on the NK cells and the HLA epitopes on the U266 cell line. Phenotypically, however, the infused NK cell population was heterogeneous because NK cells express one or a combination of inhibitory receptors on their cell surface hence leading to different NK cells subsets; i.e., subsets exclusively expressing KIRs mismatched with the HLA ligands present on U266 target cells, subsets exclusively expressing KIRs matched with the ligands and subsets expressing both types of KIRs. Therefore, only a small percentage of the infused NK cells exclusively expressed KIRs mismatched with the U266 HLA ligand (HLA-C1) and the majority of NK cells (co-)expressed NKG2A. We recently showed, that in vitro KIR-ligand mismatched NK cells are more effective than matched NK cells in mediating myeloma cell killing [22]. In addition, we demonstrated that U266 cells in this disseminated MM model expressed HLA-E at a level that is sufficient to inhibit NKG2A expressing NK cells [22]. Based on these data, NK cells expressing mismatched KIRs and lacking expression of matched KIRs or NKG2A could be expected to be the most potent effector cells against MM in vivo as well. Future experiments with HLA-E gene knock-down in U266-luc cells in the RAG2 ${ }^{-1} \gamma \mathrm{c}^{-1-}$ mice might enable us to determine the efficacy-blocking effect of HLA-E expression in NK cell therapy. Ruggeri et al., have demonstrated that, in an AML mouse model, indeed the mismatched NK cells mediated in vivo anti-tumor effects while matched NK cells did not [6]. Therefore, it is highly relevant to study whether KIR-ligand mismatched NK cells are indeed more potent against $\mathrm{MM}$ in vivo because acquiring high enough numbers of these subsets for clinical application will be challenging and it is probably worthwhile to develop strategies to promote expansion or development of NK cell subsets with the highest anti-MM reactivity.

Our data show that U266 cells are sensitive for NK cell killing in vitro (Figure 1) while in vivo these cells seemed to be more difficult to eliminate (Figure 2). An explanatory factor could be that only a fraction of the NK cells actually homes to the bone marrow resulting in a less favorable E:T ratio as compared to in vitro. Phenotypic analysis of bone marrow biopsies at the time of sacrifice of the mice revealed that activated NK cells derived from peripheral blood and cord blood indeed can home to the site of tumor location in our model (Figure 4). This is in line with a previous study showing that cord blood derived NK cells migrate to the bone marrow of NSG mice and functionally express CXCR4 [28], a chemokine receptor described to be important for homing to the bone marrow [34], and with the observation that primary blood derived NK cells express CXCR4 [35]. In addition to NK cells, we observed some $\mathrm{T}$ cells (huCD45+CD3+CD56-) and human leukocytes that were neither NK cells nor T cells (huCD45+CD3-CD56-) in mice receiving NK cells from peripheral blood. Presumably this was the result of the minor impurity of the peripheral blood derived NK cell product. Although, in principle contaminating $\mathrm{T}$ cells in the peripheral blood product can mediate an anti-MM activity as well, the actual numbers of $\mathrm{T}$ cells contaminating our cell preparations were not sufficient to explain the anti-MM response. To induce a xenogeneic GvHD response with infused PBMC cells at least $6 \times 10^{6} \mathrm{~T}$ cells are required in pre-conditioned mice (TBI + toxic liposomes) [25]. In the experiment where we combined cyclo/TBI and NK cell treatment, we did not observe any human lymphocytes in the bone marrow at the time of sacrifice, which presumably was due to the longer follow-up period 108 days vs 60 days in the experiment with activated NK cells without cyclo/TBI.

In the $\mathrm{RAG}^{-/} \gamma \mathrm{c}^{-/}$model, $\mathrm{MM}$ cells grow in their natural environment, i.e., the bone marrow, making it an excellent model for preclinical testing of immunotherapy reflecting MM pathophysiology. This is important because it is exactly this environment that, through the presence of factors like hypoxia [23] and stromal cells [36], or soluble factors like PGE2 [37] can suppress development of effective anti-tumor immunity making it extremely important to test the anti-MM capacity of NK cells in such relevant models. An interesting other pr clinical model recently developed by some members of our group is a scaffold-based model where patient derived MM cells grow on s.c. implanted scaffolds [38]. In such a model, it would be highly $i$ teresting to address the synergistic potential of a combination of a proaches targeting immunosuppressive bone marrow associated factors with alloreactive NK cell therapy to achieve maximal efficiency of the response. One such approach can be the combination of chemo/radiotherapy and activated NK cells that, in our study, resulted in a better anti-MM response as compared to treatment with activated NK cells alone. Here, we did not study the mechanism by which the conditioning regime enhanced NK cell functioning but, several previous studies demonstrated that either chemo or radiotherapy could render tumor cells more susceptible for NK cells by up regulation of activating ligands [39-41]. It can be hypothesized that donor dendritic cells are killed by the host NK cells, thus a $\mathrm{T}$ cell mediated GvHD cannot be induced [42]. On the other hand, the conditioning treatment could trigger the release of NK cell promoting cytokines such as IL-2, IL-15 and IL-21 [43] or promote recruitment or survival of the NK cells at the tumor site $[44,45]$. Also, it would be interesting to combine the immunomodulatory drug Pomalidomide or ADCC triggering agents such as Elotuzumab to boost the NK cell activity, in this model.

In the current proof-of-concept study, we showed the biological potential of alloreactive NK cells against disseminated MM. In addition, we demonstrated that IL-2 activated NK cells can home to 
Citation: Sarkar S, Noort W, Van Elssen CHMJ, Groen R, van Bloois L, et al. (2015) Alloreactive Natural Killer Cells Have Anti-Tumor Capacity Against Disseminated Human Multiple Myeloma in Rag2-/- $\gamma$ C-/-Mice When Combined With Low Dose Cyclophosphamide and Total Body Irradiation. J Clin Immunol Immunother 2: 008.

the bone marrow of MM bearing mice and that anti-MM responses occurred in a dose dependent manner. The latter suggests that infusion of a higher number of alloreactive NK cells can help to improve clinical efficacy and those patients should, preferably, be treated in remission or at least with low residual disease. Our data also demonstrate that combination therapy of alloreactive NK cells and chemo/radiotherapy could help to improve the anti-MM effect. The $\mathrm{RAG}^{-1-} \gamma \mathrm{c}^{-/-}$mouse $\mathrm{MM}$ is a pathophysiologically relevant model to further develop and optimize NK cell treatment protocols and to test ex vivo expanded/activated NK cells ultimately leading to enhanced clinical efficacy.

\section{Acknowledgements}

The $\mathrm{RAG}^{-1-} \gamma \mathrm{c}^{-/-}$mice were originally obtained from the Amsterdam Medical Center (AMC, Amsterdam, The Netherlands). The myeloma cell line LME-1 was kind gift from Dr. R. van Oers, AMC, Amsterdam, The Netherlands. The authors would like to thank the tissue-typing laboratory (MUMC+) for HLA typing of the cell lines. The authors would also like to thank Dr. Dirk Groenewegen and Dr. Jan Spanholz (Glycostem technologies, Nijmegen) and Dr. Harry Dolstra (Radboud UMC) for providing cord blood derived NK cells. The authors would also like to thank Regina de Jong-Korlaar and Frans Hofhuis (Department of Cell Biology, University Medical Center Utrecht) for their technical assistance with animal studies. L. Wieten was supported by a personal grant from Dutch Cancer association (KWF Kankerbestrijding; UM2012-5375). G. Bos, M. van Gelder and L. Wieten were supported by a grant from the Kankeronderzoeksfonds Limburg.

\section{References}

1. Raab MS, Podar K, Breitkreutz I, Richardson PG, Anderson KC (2009) Multiple myeloma. Lancet 374: 324-339.

2. Palumbo A, Anderson K (2011) Multiple myeloma. N Engl J Med 364: 10461060.

3. Bensinger WI (2009) Role of autologous and allogeneic stem cell transplantation in myeloma. Leukemia 23: 442-448.

4. Lokhorst HM, Schattenberg A, Cornelissen JJ, Thomas LL, Verdonck LF (1997) Donor leukocyte infusions are effective in relapsed multiple myeloma after allogeneic bone marrow transplantation. Blood 90: 4206-4211.

5. Shlomchik WD (2007) Graft-versus-host disease. Nat Rev Immunol 7: 340352.

6. Ruggeri L, Capanni M, Urbani E, Perruccio K, Shlomchik WD, et al. (2002) Effectiveness of donor natural killer cell alloreactivity in mismatched hematopoietic transplants. Science 295: 2097-2100.

7. Vivier E, Ugolini S, Blaise D, Chabannon C, Brossay L (2012) Targeting natural killer cells and natural killer T cells in cancer. Nat Rev Immunol 12: 239252

8. Colonna M, Borsellino G, Falco M, Ferrara GB, Strominger JL (1993) HLA-C is the inhibitory ligand that determines dominant resistance to lysis by NK1and NK2-specific natural killer cells. Proc Natl Acad Sci USA 90: 1200012004.

9. Moretta L, Locatelli F, Pende D, Marcenaro E, Mingari MC, et al. (2011) Killer Ig-like receptor-mediated control of natural killer cell alloreactivity in haploidentical hematopoietic stem cell transplantation. Blood 117: 764-771.

10. Parham P (2005) MHC class I molecules and KIRs in human history, health and survival. Nat Rev Immunol 5: 201-214.

11. Jonsson AH, Yokoyama WM (2009) Natural killer cell tolerance licensing and other mechanisms. Adv Immunol 101: 27-79.
12. Kim S, Poursine-Laurent J, Truscott SM, Lybarger L, Song YJ, et al. (2005) Licensing of natural killer cells by host major histocompatibility complex class I molecules. Nature 436: 709-713.

13. Ruggeri L, Mancusi A, Capanni M, Urbani E, Carotti A, et al. (2007) Donor natural killer cell allorecognition of missing self in haploidentical hematopoietic transplantation for acute myeloid leukemia: challenging its predictive value. Blood 110: 433-440.

14. Osterborg A, Nilsson B, Björkholm M, Holm G, Mellstedt H (1990) Natural killer cell activity in monoclonal gammopathies: relation to disease activity. Eur J Haematol 45: 153-157.

15. Frohn C, Höppner M, Schlenke P, Kirchner H, Koritke P, et al. (2002) Anti-myeloma activity of natural killer lymphocytes. Br J Haematol 119: 660-664.

16. Carbone E, Neri P, Mesuraca M, Fulciniti MT, Otsuki T, et al. (2005) HLA class I, NKG2D, and natural cytotoxicity receptors regulate multiple myeloma cell recognition by natural killer cells. Blood 105: 251-258.

17. El-Sherbiny YM, Meade JL, Holmes TD, McGonagle D, Mackie SL, et al. (2007) The requirement for DNAM-1, NKG2D, and NKp46 in the natural killer cell-mediated killing of myeloma cells. Cancer Res 67: 8444-8449.

18. Shi J, Tricot G, Szmania S, Rosen N, Garg TK (2008) Infusion of haplo-identical killer immunoglobulin-like receptor ligand mismatched NK cells for relapsed myeloma in the setting of autologous stem cell transplantation. $\mathrm{Br} \mathrm{J}$ Haematol 143: 641-53.

19. Szmania S, Lapteva N, Garg T, Greenway A, Lingo J, et al. (2015) Ex vivo-expanded natural killer cells demonstrate robust proliferation in vivo in high-risk relapsed multiple myeloma patients. J Immunother 38: 24-36.

20. Katodritou E, Terpos E, North J, Kottaridis P, Verrou E, et al. (2011) Tumor-primed natural killer cells from patients with multiple myeloma lyse autologous, NK-resistant, bone marrow-derived malignant plasma cells. Am J Hematol 86: 967-973.

21. Kröger N, Shaw B, lacobelli S, Zabelina T, Peggs K, et al. (2005) Comparison between antithymocyte globulin and alemtuzumab and the possible impact of KIR-ligand mismatch after dose-reduced conditioning and unrelated stem cell transplantation in patients with multiple myeloma. $\mathrm{Br} \mathrm{J}$ Haematol 129: 631-643.

22. Sarkar S, van Gelder M, Noort W, Xu Y, Rouschop KM, et al. (2015) Optimal selection of natural killer cells to kill myeloma: the role of HLA-E and NKG2A. Cancer Immunol Immunother 64: 951-963.

23. Sarkar S, Germeraad WT, Rouschop KM, Steeghs EM, van Gelder M, et al (2013) Hypoxia induced impairment of NK cell cytotoxicity against multiple myeloma can be overcome by IL-2 activation of the NK cells. PLoS One 8: 64835 .

24. van Rijn RS, Simonetti ER, Hagenbeek A, Hogenes MC, de Weger RA (2003) A new xenograft model for graft-versus-host disease by intravenous transfer of human peripheral blood mononuclear cells in RAG2-/- gammac-/- double-mutant mice. Blood 102: 2522-2531.

25. Rozemuller H, van der Spek E, Bogers-Boer LH, Zwart MC, Verweij V, et al. (2008) A bioluminescence imaging based in vivo model for preclinical testing of novel cellular immunotherapy strategies to improve the graft-versus-myeloma effect. Haematologica 93: 1049-1057.

26. Spanholtz J, Preijers F, Tordoir M, Trilsbeek C, Paardekooper J, et al. (2011) Clinical-grade generation of active NK cells from cord blood hematopoietic progenitor cells for immunotherapy using a closed-system culture process. PLoS One 6: 20740.

27. Spanholtz J, Tordoir M, Eissens D, Preijers F, van der Meer A, et al. (2010) High log-scale expansion of functional human natural killer cells from umbilical cord blood CD34-positive cells for adoptive cancer immunotherapy. PLoS One 5: 9221

28. Cany J, van der Waart AB, Tordoir M, Franssen GM, Hangalapura BN, et al. (2013) Natural killer cells generated from cord blood hematopoietic progenitor cells efficiently target bone marrow-residing human leukemia cells in NOD/ SCID/IL2Rg (null) mice. PLoS One 8: 64384. 
Citation: Sarkar S, Noort W, Van Elssen CHMJ, Groen R, van Bloois L, et al. (2015) Alloreactive Natural Killer Cells Have Anti-Tumor Capacity Against Disseminated Human Multiple Myeloma in Rag2-/- $\gamma$ C-/-Mice When Combined With Low Dose Cyclophosphamide and Total Body Irradiation. J Clin Immunol Immunother 2: 008.

29. Frings PW, Van Elssen CH, Wieten L, Matos C, Hupperets PS, et al. (2011) Elimination of the chemotherapy resistant subpopulation of $4 \mathrm{~T} 1$ mouse breast cancer by haploidentical NK cells cures the vast majority of mice. Breast Cancer Res Treat 130: 773-781.

30. Sutlu T, Alici E (2009) Natural killer cell-based immunotherapy in cancer: current insights and future prospects. J Intern Med 266: 154-181.

31. Alici E, Konstantinidis KV, Sutlu T, Aints A, Gahrton G, et al. (2007) Anti-myeloma activity of endogenous and adoptively transferred activated natural killer cells in experimental multiple myeloma model. Exp Hematol 35: 18391846.

32. Garg TK, Szmania SM, Khan JA, Hoering A, Malbrough PA, et al. (2012) Highly activated and expanded natural killer cells for multiple myeloma immunotherapy. Haematologica 97: 1348-1356.

33. Swift BE, Williams BA, Kosaka Y, Wang XH, Medin JA (2012) Natural kille cell lines preferentially kill clonogenic multiple myeloma cells and decrease myeloma engraftment in a bioluminescent xenograft mouse model. Haematologica 97: 1020-1028.

34. Beider K, Nagler A, Wald O, Franitza S, Dagan-Berger M, et al. (2003) Involvement of CXCR4 and IL-2 in the homing and retention of human NK and NK T cells to the bone marrow and spleen of NOD/SCID mice. Blood 102 1951-1958.

35. Inngjerdingen M, Damaj B, Maghazachi AA (2001) Expression and regulation of chemokine receptors in human natural killer cells. Blood 97: 367-375.

36. McMillin DW, Delmore J, Negri JM, Vanneman M, Koyama $S$, et al. (2012) Compartment-Specific Bioluminescence Imaging platform for the high-throughput evaluation of antitumor immune function. Blood 119: 131 138

37. Van Elssen $\mathrm{CH}$, Vanderlocht J, Oth T, Senden-Gijsbers BL, Germeraad WT, et al. (2011) Inflammation-restraining effects of prostaglandin E2 on Natura Killer-Dendritic Cell (NK-DC) interaction are imprinted during DC maturation. Blood 118: 2473-2482.
38. Groen RW, Noort WA, Raymakers RA, Prins HJ, Aalders L, et al. (2012) Reconstructing the human hematopoietic niche in immunodeficient mice: opportunities for studying primary multiple myeloma. Blood 120: 9-16

39. Fionda C, Soriani A, Malgarini G, lannitto ML, Santoni A, et al. (2009) Heat shock protein-90 inhibitors increase $\mathrm{MHC}$ class I-related chain $A$ and $B$ ligand expression on multiple myeloma cells and their ability to trigger NK cell degranulation. J Immunol 183: 4385-4394.

40. Shi J, Tricot GJ, Garg TK, Malaviarachchi PA, Szmania SM, et al. (2008) Bortezomib down-regulates the cell-surface expression of HLA class I and enhances natural killer cell-mediated lysis of myeloma. Blood 111: 1309-1317.

41. Wu X, Shao Y, Tao Y, Ai G, Wei R, et al. (2011) Proteasome inhibitor lactacystin augments natural killer cell cytotoxicity of myeloma via downregulation of HLA class I. Biochem Biophys Res Commun 415: 187-192.

42. Laffont S, Seillet C, Ortaldo J, Coudert JD, Guéry JC (2008) Natural killer cells recruited into lymph nodes inhibit alloreactive T-cell activation through perforin-mediated killing of donor allogeneic dendritic cells. Blood 112: 661 671

43. Bracci L, Moschella F, Sestili P, La Sorsa V, Valentini M, et al. (2007) Cyclophosphamide enhances the antitumor efficacy of adoptively transferred immune cells through the induction of cytokine expression, B-cell and T-cell homeostatic proliferation, and specific tumor infiltration. Clin Cancer Res 13 644-653.

44. Sharabi A, Ghera NH (2010) Breaking tolerance in a mouse model of multiple myeloma by chemoimmunotherapy. Adv Cancer Res 107: 1-37.

45. Sharabi A, Haran-Ghera N (2011) Immune recovery after cyclophosphamide treatment in multiple myeloma: implication for maintenance immunotherapy. Bone Marrow Res 2011: 269519. 